\title{
Research on the performance testing method of GNSS receiver
}

\author{
Xiaofen $\mathrm{Hu}^{\mathrm{a}}$, Zhihong Qiao, Chunyang Zhang \\ Research Institute of Chemical Defense, Peking, 102205, China \\ aEmail: fen9155@163.com
}

Keywords: satellite signal simulator; performance testing; receiver

\begin{abstract}
With the continuous development of the global navigation satellite system (GNSS) and related technologies, the research of performance testing of navigation receiver has gradually become the focus attention of the satellite navigation industry. Due to the influence of environmental factors, repeatability of traditional testing methods is poor and is difficult to ensure accuracy. Receiver performance testing methods based on GNSS signal simulator is using of satellite navigation signal simulator to simulate real satellite signals and provide a stable and accuracy testing environment for GNSS receiver performance testing. This paper focuses on testing methods for GNSS receiver and provides a full range of test environment for the development and use of the receiver.
\end{abstract}

\section{Introduction}

GNSS is short for Global Navigation Satellite System. In recent decades, with the continuous development of the global navigation satellite system (GNSS) and related technologies, especially the construction and development of GPS of the United States, GLONASS of the Russian, GALILEO of the EU and BDS of China, receiver of the GNSS has been applied increasingly, the research of performance testing of navigation receiver has gradually become the focus attention of the satellite navigation industry.

The traditional testing method is using an antenna to receive the actual satellite signals, but it is easy to influence by the complexity of the weather changes, ionospheric delay, multipath effects and other environmental factors. So the measurement repeatability is very poor , the test accuracy is difficult to guarantee, and can't be tested according to the needs of users, who do any change to meet the needs of various signal parameters of various test scenarios. Based on the above drawbacks, GNSS signal simulator comes into being, it can work on different scenarios which were simulated GNSS receiver according to the different needs of users, simulate different GNSS signal under different working conditions and create the conditions for testers to test and validate the performance of work under different environmental scenarios. The advantages of GNSS signal simulator is simulating GNSS signals in limited space conditions on a number of different work environments, so that the required time and the cost of receiver performance testing has been greatly reduced.

\section{Classification of GNSS receivers}

\section{Divided by the purpose of receivers}

\section{(1) navigation receivers}

This type of navigation receiver is mainly used for motion vectors, which gives real-time position and velocity vector. The receivers generally use the $\mathrm{C} / \mathrm{A}$ code pseudo-range measurements, low single-point real-time positioning accuracy, typically $\pm 25 \mathrm{~m}$, cheap, widely used. According to different application areas, the receivers can be further divided into: Vehicle type - for vehicle navigation and positioning; nautical type - for ship navigation and positioning; aviation type - used in aircraft navigation and positioning. As the aircraft running speed, on the use of aviation receivers can adapt to the requirements of high-speed movement. Spaceborne type - for satellite navigation and positioning. As the speed of the satellite up $7 \mathrm{~km} / \mathrm{s}$, the requirements of the receiver is much higher. 


\section{(2) geodetic receivers}

Geodetic receivers mainly are used for precision geodetic measurements and precision engineering, high positioning accuracy. The structure of instrument is complex and expensive.

\section{Divided by the carrier frequency of the receivers}

(1) single-frequency receiver

Single-frequency receiver can only receive a single frequency carrier signal, test the measured carrier phase observations to position. Because it is not effectively to eliminate the influence of ionospheric delay, single-frequency receivers can only apply in short baseline (less than 15km) in precise positioning.

(2) dual-frequency receiver

Dual-frequency receiver can simultaneously receive two frequency carrier signal.

Using different time of dual-frequency ionospheric delay, you can eliminate the effects of ionospheric delay of electromagnetic signals. So dual-frequency receivers can be used for thousands of kilometers in the precise positioning.

\section{Test Principle}

In recent years with GNSS signal simulator widely used, using of GNSS signal simulator and GNSS receiver to build test platform become a common means of testing receiver performance. The test platform of GNSS receiver simulation in this paper include four parts, GNSS signal simulator, GNSS receiver, performance testing software and data processing software . GNSS signal simulator provides analog signals of satellite navigation receiver for processing. Performance testing software adjusts the parameters of the navigation signal to meet the specific performance requirements, through startup and shutdown of controlling GNSS receivers to implement testing process for multiple times. During the testing process, the corresponding parameters from the signal simulator and the actual measured values of the receiver get together into the data processing software for data processing, show in the way of coordinate curve and compare with the performance of the theoretical curve to achieve the GNSS receiver performance evaluation.

Workflow of the GNSS receiver test platform shown in figure 3-1:

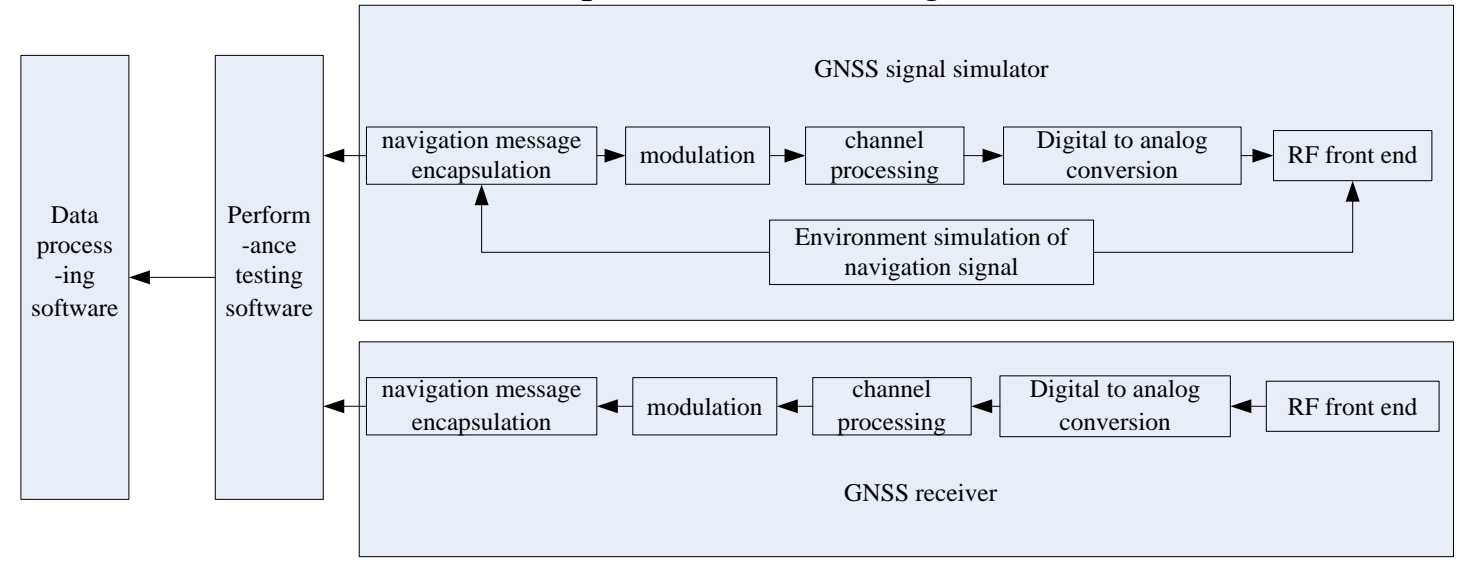

Figure 3-1, in the performance testing process of GNSS receiver, first step is setting test performance, test parameters and test environment by performance testing software, and then the signal parameters of signal simulator is determined according to the environmental parameters, generates analog GNSS signal for the receiving test, completes the performance testing process. Finally the test data is processed by the data processing software.

Works of the GNSS receiver test platform shown in figure 3-2: 


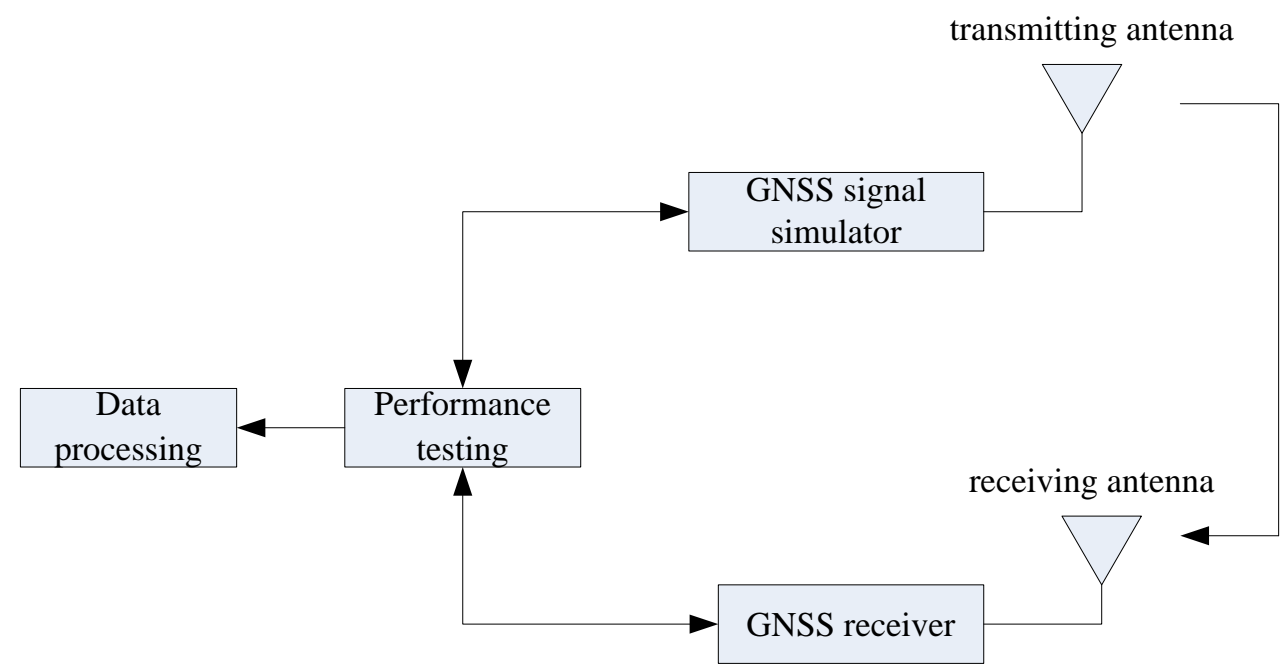

Figure 3-2, the test platform control GNSS signal simulator to produce the navigation signals of meeting the test requirements, emit the signal through a transmit antenna. GNSS receivers receive and process the signal. During the testing process, the test software obtains the data processing parameters from the signal simulator and the receiver. The final test result is obtained.

\section{Test Methods}

\section{Test Methods}

Receiver performance testing is a test behavior of the performance index of GNSS receiver to evaluate the performance of GNSS receiver synthetically. Evaluation and judgment can be made on the overall performance test of the receiver by testing activities to determine whether they meet the requirements of practical applications. Receiver performance testing is generally based on the test platform which are consisted of GNSS signal simulator and GNSS receivers. The main indicators of performance tests are including time to first fix test, recapture time test, the receiver sensitivity test and navigation accuracy test.

(1) the testing of time to first fix (TTFF)

First, according to the testing requirements for GNSS signal simulator and receiver, we initially set to start in a different way (cold start, warm start) under different settings: In the cold start, receiver ephemeris needs to be emptied. In the warm start, ephemeris data needs to be input into the receiver. and then the receiver captures the signal. The software records the time from start to outputting navigation results. That is, the value of the income measure TTFF. Repeating the above process we can obtain a plurality of sets of data, and sent to the data processing section for data processing.

(2) the testing of time to recapture

First, according to the testing requirements for GNSS signal simulator and receiver, we initially set. Signal simulator emits GNSS navigation signals. Start the receiver until the receiver keeps track of the state. When the receiver output the navigation results, close the signal simulator , and then the receiver lose lock. Restarting the simulator, the software records the time from restart to outputting navigation results. That is the time to recapture the resulting measurement values. Repeat the above process we can obtain a plurality of sets of data, and sent to the data processing section for processing.

(3) the test of receiver sensitivity

First, according to the testing requirements for GNSS signal simulator and receiver, we initially set. Due to the different requirements of sensitivity for capturing and tracking sensitivity testing, we adjust the output power of the signal simulator to get close to the theoretical acquisition sensitivity and tracking sensitivity. In the test of sensitivity for capturing, signal simulator emits GNSS navigation signals. The receiver captures the signal. If the receiver capture successfully, turn off the 
receiver, reduce the signal power of the simulator, restart the receiver to capture the signal. Repeat the above steps until the receiver can not capture, record the output signal power. That is the sensitivity of the resulting values. In the test of sensitivity for tracking, signal simulator emits GNSS navigation signals. If the receiver track successfully, reduce the signal power of the simulator until the receiver loses lock of the signal, then record the output signal power of the simulator. That is the tracking sensitivity values of this time .

Repeat the above process we can obtain a plurality of sets of data, and sent to the data processing section for processing.

(4) the test of positioning accuracy

First, according to the testing requirements for GNSS signal simulator and receiver, we initially set motion state of the receiver to test the positioning accuracy in the different motion states. Signal simulator emits GNSS navigation signals. Start the receiver, when the navigation results to be extracted, the receiver output the current pseudo observations. Keep the tracking state, get multiple sets of pseudo-range observations, seeking its variance can be obtained under the current signal carrier to noise ratio of the pseudo-range measurement error. In different signal carrier to noise ratio, the above process is repeated to obtain a plurality of sets of data, and the data is sent to processing section for processing.

\section{Data Processing}

After obtaining the test data, we need to do data processing, obtain the performance curve of GNSS receiver and compare with the theoretical curve to evaluate the performance of receiver.

(1) the testing of time to first fix (TTFF)

The arithmetic mean of multiple sets of the measured TTFF values is:

$$
\overline{\mathrm{T}}=\frac{1}{n} \sum_{\mathrm{i}=1}^{\mathrm{n}} T_{\mathrm{i}}
$$

$T_{\tilde{i}}$ is the TTFF values of the $i$ times. $n$ is the total number of measurements.

The standard deviation of TTFF values is:

$$
S=\sqrt{\frac{1}{n-1} \sum_{i=1}^{n}\left(T_{i}-\bar{T}\right)^{2}}
$$

The range of TTFF values is taken as $\overline{\mathrm{T}} \pm 2 \mathrm{~S}$.

(2) the testing of time to recapture

The arithmetic mean of multiple sets of the recapture time is:

$$
\overline{\mathrm{t}}=\frac{1}{\mathrm{n}} \sum_{\mathrm{i}=1}^{\mathrm{n}} t_{\mathrm{i}}
$$

$t_{i}$ is the recapture time of the $i$ times. $n$ is the total number of measurements.

The standard deviation of recapture time is:

$$
S=\sqrt{\frac{1}{n-1} \sum_{i=1}^{m}\left(t_{i}-\bar{t}\right)^{2}}
$$

The range of recapture time is taken as $\bar{t} \pm 2 S$.

(3) the test of receiver sensitivity

The arithmetic mean of multiple sets of the receiver sensitivity (the lowest capture / tracking power values) is:

$$
\overline{\mathrm{P}}=\frac{1}{\mathrm{n}} \sum_{\mathrm{i}=1}^{\mathrm{n}} P_{\mathrm{i}}
$$

$P_{i}$ is the lowest capture / tracking power values of the $\mathrm{i}$ times. $\mathrm{n}$ is the total number of measurements.

The standard deviation of the lowest capture / tracking power values is:

$$
S=\sqrt{\frac{1}{n-1} \sum_{i=1}^{n}\left(P_{i}-\bar{P}\right)^{2}}
$$

The range of the receiver sensitivity is taken as $\overline{\mathrm{P}} \pm 2 \mathrm{~S}$.

(4) the test of positioning accuracy

The arithmetic mean of the pseudorange observations is:

$$
\overline{\mathrm{D}}=\frac{1}{n} \sum_{i=1}^{n} D_{\mathrm{i}}
$$

$D_{i}$ is the pseudorange observations of the i times. $n$ is the total number of measurements.

The standard deviation of the pseudorange observations is: 


$$
\mathrm{D}_{p r}=\frac{1}{n-1} \sum_{i=1}^{n}\left(D_{i}-\bar{D}\right)^{2}
$$

The error of the pseudo-range observations is $\mathrm{D}_{p r}$.

\section{Conclusion}

This paper presents a test method of receiver performance based on a navigation signal simulator. It is not only to achieve the performance testing of the receiver, but also to provide a new means of testing for receiver and a reference for manufacturers and metrological verification institutions.

\section{Referencess}

[1] Liu Zhiguo. Research and implementation of signal simulator of navigation satellite[D]. Taiyuan: North Central University, 2014. [2] Jia Guangchao, Xiao Haixia. Performance test and analysis of the Beidou satellite based on signal simulator [J]. Navigation and positioning of the Journal, 2013(1) 04: 14-16.

[3] Li Haifeng. Research on test method and scene design of navigation satellite[D]. Zhengzhou: The PLA Information Engineering University, 2008.

[4] Zhai Jianwei, Wang Dangwei, Lv Hongli. Analysis of receiver function and performance index of high precision navigation satellite [C]. CSNC2013 The fourth session of the Chinese Satellite Navigation Conference, 2013.

[5] Lou Yidong, Ao Shuijin, Nie Jugen. Performance analysis of GPS receiver for vehicle navigation [J]. Surveying and spatial information, 2009(32)04: 5-8.

[6] Liao Chaoming, Tan Qinglin, Qin Yuanli. Discussion on the GPS receiver verification principle and automatic processing of calibration data [J]. Bulletin of Surveying and mapping, 2007(32): 1113. 